\title{
The pause that refreshes, or does it? Mechanisms in torsades de pointes
}

Dessertenne, who coined the term "torsades de pointes", was one of the first to draw attention to the association of long QT related arrhythmia (commonly caused by heart block or drugs) and underlying bradycardia. ${ }^{1}$ Indeed, the "short-long-short" series of cycles before torsade de pointes is so characteristic of acquired "long QT syndrome" (LQTS) that lack of a "pause" before onset calls into question the diagnosis. ${ }^{2}{ }^{3}$ Some features of the congenital LQTS are very similar to those in the acquired form. These include a preponderance of women among patients who are symptomatic with syncope or sudden death, and the possible potentiating role of hypokalaemia in the genesis of the arrhythmia. Indeed, extraordinary advances in our understanding of the molecular basis of the congenital and acquired syndromes now point to common mechanisms that underlie these two closely related entities. ${ }^{45}$ However, despite considerable attention to the electrocardiographic features of these diseases, it is not known whether the onset of the arrhythmia in the congenital form is pause dependent as in the acquired form. In a recent issue of Heart, Visken and colleagues summarised the electrocardiographic features at onset of torsade de pointes in patients with the congenital form of the syndrome. ${ }^{6}$ Their findings - that not all episodes are pause dependent - provide intriguing new data for investigators interested in linking the clinical and molecular events involved in genesis of long QT related arrhythmias.

\section{Mutations causing the congenital LQTS}

Congenital LQTS remained an electrophysiologic curiosity until 1995 and 1996, when Keating and colleagues linked the syndrome to mutations in the genes encoding ion channel proteins. These findings have had important implications for our understanding not only of arrhythmia mechanisms in this obscure but interesting disease, but also in further understanding the concept of dysregulated expression or function of these proteins as a proximate cause of arrhythmias in common clinical settings as diverse as atrial fibrillation and congestive heart failure.

Five loci in the human genome have been linked to the LQTS in different families (table 1). At four of these, specific genes, and mutations in those genes, have been identified; no disease gene has been identified for the LQT4 locus, and the LQT6 locus has been associated with (but not yet formally linked to) drug induced LQTS. In each disease gene identified to date, multiple mutations have been reported in different families. There is now a considerable body of evidence that the clinical presentation in these families is determined by the specific disease gene, and perhaps by the specific mutation. Thus, for example, cardiac events (syncope or sudden death) occur with emotional or physical stress in the vast majority of patients with mutations in KvLQT1, whereas most events occur at night in patients with mutations in SCN5A. The classical "startle" response to auditory stimuli appears relatively specific for mutations in HERG. ${ }^{7}$ One of the most important findings to emerge from ongoing clinical-genetic studies is the identification of patients with normal QT intervals who harbour LQTS mutations. ${ }^{8}$ This "incomplete penetrance" of the phenotype has important implications for use of antiarrhythmic drugs and perhaps even for understanding susceptibility to sudden death in otherwise asymptomatic individuals.

In theory, prolongation of the cardiac action potential resulting in QT prolongation on the surface ECG can be accomplished by either a decrease in outward (repolarising) currents, generally carried by potassium ions, or an increase in inward (depolarising) current, generally carried by sodium or calcium ions. Molecular and cellular studies of the long QT syndromes have revealed both types of defects. Mutations affecting the protein subunits that make up the outward (repolarising) potassium currents $\mathrm{I}_{\mathrm{Ks}}$ and $\mathrm{I}_{\mathrm{Kr}}$ probably do so by reducing these currents. Mutations affecting the cardiac sodium channel result in a small increase in inward current during the action potential plateau, thereby prolonging cardiac action potentials. An extraordinarily fruitful area of basic biophysical research is elucidation of the multiple cellular and molecular mechanisms that produce these effects. As well, it is now appreciated that the vast majority of drugs implicated in acquired LQTS are blockers of $\mathrm{I}_{\mathrm{Kr}}$, thereby providing the mechanistic link between the congenital and the acquired syndromes. Mutations in KvLQT1 and HERG, affecting $\mathrm{I}_{\mathrm{Ks}}$ and $I_{\mathrm{Kr}}$ respectively, are the most common causes of congenital LQTS, accounting for perhaps $90 \%$ of cases. Thus, studies describing clinical findings in unselected,

Table 1 Congenital long QT syndromes

\begin{tabular}{|c|c|c|c|c|c|c|}
\hline & Gene* & Current & Frequency & $E C G$ & Circumstances surrounding events & $D Q T$ with exercise \\
\hline LQT1 & $K v L Q T 1$ & $\downarrow \mathrm{I}_{\mathrm{Ks}}$ & $40 \%$ & Broad T & Exercise, emotion & - \\
\hline LQT2 & $H E R G$ & $\downarrow \mathrm{I}_{\mathrm{Kr}}$ & $40 \%$ & Bifid T & Sudden auditory stimuli & Normal \\
\hline LQT3 & SCN5A & $\uparrow \mathrm{I}_{\mathrm{Na}}$ & $10 \%$ & Long isoelectric ST & Sleep & Supernormal \\
\hline LQT4 & Unknown & & Rare & & & \\
\hline LQT5 & $\min K$ & $\downarrow \mathrm{I}_{\mathrm{Ks}}$ & Rare & & & \\
\hline LQT6 & $M i R P 1$ & $\downarrow \mathrm{I}_{\mathrm{Kr}}(?)$ & Rare & & & \\
\hline JLN1 & $K v L Q T 1$ & $\downarrow \mathrm{I}_{\mathrm{Ks}}$ & Rare & Marked $\uparrow Q T$ & Exertion & \\
\hline JLN2 & $\min \widehat{K}$ & $\downarrow \mathrm{I}_{\mathrm{Ks}}$ & Rare & Marked $\uparrow \mathrm{QT}$ & Exertion & \\
\hline
\end{tabular}

LQT1-6 are autosomal dominant without deafness (also termed Romano-Ward syndrome). JLN: Jervell-Lange-Neilsen syndrome (autosomal recessive with deafness) arises in individuals who inherit abnormal alleles from both parents, and is much rarer.

*Some of these genes have alternate names. The "official" terms for KvLQT1, HERG, $\operatorname{minK}$, and MiRP1 are KCNQ1, KCNH2, KCNE1, and KCNE2, respectively. MinK is also known as IsK. HERG is an acronym for "Human Ether a-go-go-Related Gene", and MiRP1 is an acronym for "MinK-Related Protein -1". 


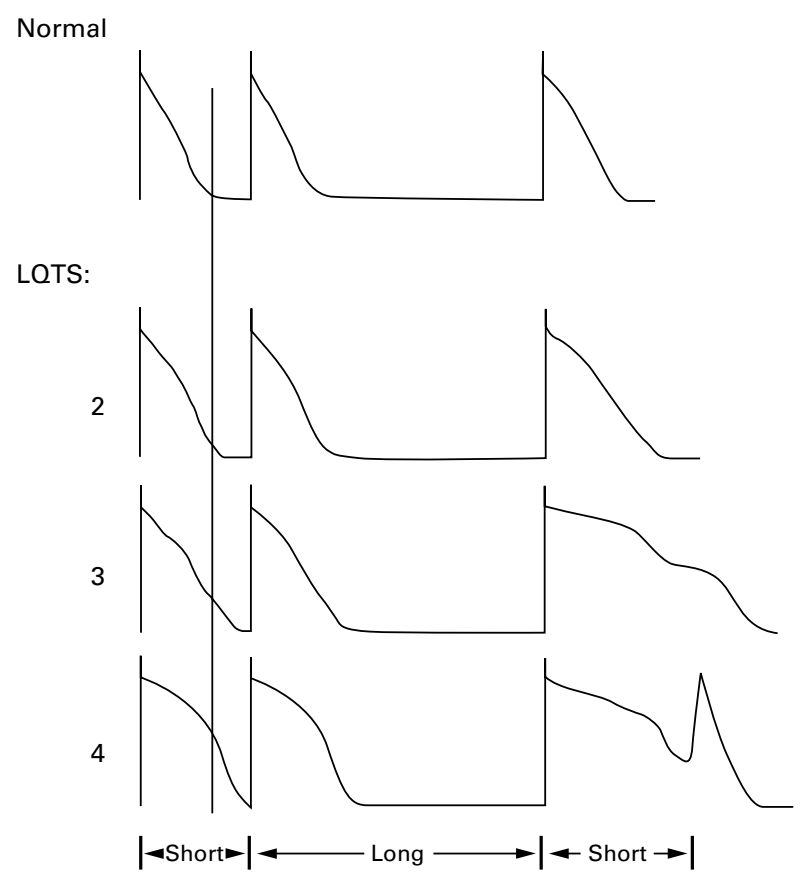

Figure 1 Schematic of cardiac action potentials before and during a pause. A normal response is shown in the top panel, and three action potentials (2,3, and 4) in long QT syndrome (LQTS) below. Action potentials in LQTS are prolonged compared to normal, indicated by the vertical line. However, after the pause, the extent of action potential prolongation is exaggerated and highly heterogeneous, with an afterdepolarisation in the third panel and a triggered upstroke in the fourth. Under these conditions, the triggered beat may propagate to sites with short action potentials (for example, action potential 2) but not to sites with longer ones (for example, action potential 3), initiating the unstable re-entry that is thought to underlie torsades de pointes. ${ }^{17} \mathrm{~A}$

"short-long-short" series of cycles would appear on the surface ECG.

ungenotyped patients with the congenital long QT syndrome (as in the series presented by Visken ${ }^{6}$ ) generally represent a mix of KvLQT1 and HERG-related familiesthat is, those in whom an adrenergic trigger seems especially prominent.

\section{Arrhythmia mechanisms in LQTS}

While these mutations provide a very nice explanation for QT prolongation, they do not, in fact, explain the development of the arrhythmias. Many lines of evidence now support the concept that, when action potentials are prolonged, inward currents through genetically normal and drug unmodified channels are recruited. These inward currents further prolong action potentials and provide secondary upstrokes in action potential configuration, explaining the early afterdepolarisations (EADs) and triggered activity that are thought to underlie initiation of torsade de pointes. The exact nature of these inward arrhythmogenic currents is under study and a number of candidates ( $\mathrm{L}$ or $\mathrm{T}$ type calcium channels, arrhythmogenic transient inward current, sodium-calcium exchange, or sodium current) have been proposed. These arrhythmogenic changes in action potential configuration probably do not occur in all cells in the myocardium. Thus, premature beats (generally arising from EAD related triggered activity) may propagate in some sites with short action potentials and block in others, where action potentials are long (fig 1). In this way, triggered activity may generate re-entrant excitation which is currently thought to underlie the maintenance of torsade de pointes.

\section{Pause dependence and reverse use dependence}

Most drugs that prolong the QT interval do so more at slow rates than at fast ones, a phenomenon that has been termed "reverse use dependence". ${ }^{9}$ This property of exaggerated QT prolongation at slow rates probably contributes to the bradycardia dependence of torsade de pointes. This observation prompted speculation that drugs prolonging the QT interval more at fast rates than at slow ones would be preferable as antiarrhythmics, since they would lack this propensity to cause torsade de pointes. Indeed, the identification of $\mathrm{I}_{\mathrm{Kr}}$ and $\mathrm{I}_{\mathrm{Ks}}$ as separate currents in the late 1980 s led to the concept that $\mathrm{I}_{\mathrm{Ks}}$ block would be one mechanism whereby "positive use dependence" could be developed as an antiarrhythmic strategy. Efforts along these lines were mounted by at least three major pharmaceutical companies, and all came to a very rapid halt with the identification of mutations in the genes encoding $\mathrm{I}_{\mathrm{Ks}}$ as a common cause of the congenital syndrome. What, then, was the problem with this formulation? The answer to this question is not completely in, but several tantalising pieces of evidence are now available to indicate that action potential prolongation at fast rates (as would be expected with either drug block of $\mathrm{I}_{\mathrm{Ks}}$ or congenital lesions reducing $\mathrm{I}_{\mathrm{Ks}}$ ) is highly arrhythmogenic, and intracellular calcium $\left(\left[\mathrm{Ca}^{2+}\right]_{\mathrm{I}}\right)$ loading, facilitated by rapid antecedent rates, maybe a crucial contributor. For example, we have shown that the magnitude of the $\left[\mathrm{Ca}^{2+}\right]_{\mathrm{I}}$ transient is enhanced by voltage clamp commands minimising a long action potential. ${ }^{10}$ Under these conditions, a calcium activated, arrhythmogenic inward current is elicited. Similarly, the Antzelevitch laboratory has demonstrated "acceleration dependent" afterdepolarisations, ${ }^{11}$ and dependence of $T$ wave alternans and $U$ waves on raised $\left[\mathrm{Ca}^{2+}\right]_{\mathrm{I}}$ in in vitro LQTS models. ${ }^{12}$

\section{Arrhythmia onsets in congenital LQTS}

Visken and colleagues found that most adults with the congenital long QT syndrome have pause dependence, as in the drug induced form. ${ }^{6}$ However, in children-who often have a much more severe form of the disease-the onset of the arrhythmia is typically not pause dependent. They speculate that the pause serves to prime the myocardium to generate torsade de pointes and that in patients with a particularly severe form of the disease, such priming may not be necessary to generate the arrhythmias. We agree with this concept, and suggest the framework outlined in fig 1. Many conditions prolong baseline action potentials in diverse settings such as the congenital syndromes, in women, or in congestive heart failure. Under these conditions, one important consequence of a rapid heart rate (as reported just before torsade de pointes in atrial fibrillation ${ }^{13}$ and in the drug induced arrhythmia ${ }^{14}$ ) would be an exaggerated elevation of $\left[\mathrm{Ca}^{2+}\right]_{\mathrm{I}}$. Importantly, the consequences of such an elevation would be most evident after a pause. It is well recognised that under normal conditions, cardiac muscle exhibits the phenomenon of post-rest potentiation, where an optimally timed pause results in augmented contraction, likely reflecting the time dependence of $\left[\mathrm{Ca}^{2+}\right]_{\mathrm{I}}$ movement into a releasable compartment in the sarcoplasmic reticulum. ${ }^{15}{ }^{16}$ Hence, with a background of long action potentials and rapid rates, a pause and its associated $\left[\mathrm{Ca}^{2+}\right]_{\mathrm{I}}$ translocations would provide an ideal breeding ground for afterdepolarisations, triggering, and heterogeneity of action potential durations that are thought to underlie torsade de pointes. Such a unifying mechanism would also nicely account for $T$ wave alternans and $U$ waves that presage torsade de pointes and are also linked to $\left[\mathrm{Ca}^{2+}\right]_{\mathrm{I}}$ oscillations. ${ }^{12}$ In summary, we view a major consequence of the first short cycle(s) of the "short-long-short" series as calcium loading. The long cycle then serves to maximise heterogeneity among neighbouring action potential durations, and in particular to allow action potentials to become sufficiently 
long in some regions of the heart to generate EADs and triggered activity. Propagation of this activity to some areas, and not to others, then initiates an episode of torsade de pointes. Notably, this formulation suggests - and the clinical data of Visken and colleagues ${ }^{6}$ support the idea-that in some patients the substrate may be sufficiently abnormal that priming conditions alone (for example, tachycardia) may elicit the arrhythmia without the pause. Clearly, these concepts have a long way to go before they can be viewed as proven. Nevertheless, clinical reports such as those from Visken and colleagues serve to reinforce the importance of the clinical investigator in providing crucial links to allow our understanding of basic mechanisms of arrhythmogenesis to go forward.

DAN M RODEN

Departments of Medicine and Pharmacology, MARK E ANDERSON

Vanderbilt University School of Medicine,

532 Medical Research Building - I,

Nashville, TN 37232-6602,

USA

email:Dan.Roden@MCMail.Vanderbilt.edu

1 Dessertenne F. La tachycardie ventriculaire à deux foyers opposés variables. Arch Mal Coeur 1966;59:263-72.

2 Kay GN, Plumb VJ, Arciniegas JG, et al. Torsades de pointes: the long-short initiating sequence and other clinical features: observations in 32 patients. 7 Am Coll Cardiol 1983;2:806-17.

3 Roden DM, Woosley RL, Primm RK. Incidence and clinical features of the quinidine-associated long QT syndrome: implications for patient care. $A m$ Heart $\mathcal{F}$ 1986;111:1088-93.
4 Roden DM, Lazzara R, Rosen MR, et al, for the SADS Foundation Task Force on LQTS. Multiple mechanisms in the long QT syndrome: current knowledge, gaps, and future directions. Circulation 1996;94:1996-2012.

5 Roden DM, Spooner PM. Inherited long QT syndromes. 7 Cardiovasc Electrophysiol 2000 In press.

6 Viskin S, Fish R, Zeltser D, et al. Arrhythmias in the congenital long QT syndrome: how often is torsades de pointes pause-dependent? Heart 2000; 83:661-6.

7 Wilde AA, Jongbloed RJ, Doevendans PA, et al. Auditory stimuli as a trigger for arrhythmic events differentiate HERG-related (LQTS2) patients from for arrhythmic events differentiate HERG-related (LQTS2) patients from

8 Priori SG, Napolitano C, Schwartz PJ. Low penetrance in the long-QT Priori SG, Napolitano C, Schwartz PJ. Low penetrance
syndrome: clinical impact. Circulation 1999;99:529-33.

9 Hondeghem LM, Snyders DJ. Class III Antiarrhythmic agents have a lot of potential, but a long way to go: reduced effectiveness and dangers of reverse use- dependence. Circulation 1990;81:686-90.

10 Wu Y, Roden DM, Anderson ME. Calmodulin kinase inhibition prevents development of the arrhythmogenic transient inward current. Circ Res 1999;84:906-12.

11 Burashnikov A, Antzelevitch C. Acceleration-induced action potential prolongation and early afterdepolarizations. $\mathcal{F}$ Cardiovasc Electrophysiol 1998;9: 934-48.

12 Shimizu W, Antzelevitch C. Cellular and ionic basis for T-wave alternans under long-QT conditions. Circulation 1999;99:1499-507.

13 Choy AMJ, Darbar D, Dell'Orto S, et al. Increased sensitivity to QT prolonging drug therapy immediately after cardioversion to sinus rhythm. $\mathcal{F}$ Am Coll Cardiol 1999;34:396-401.

14 Locati EH, Maison-Blanche P, Dejode P, et al. Spontaneous sequences of onset of torsade de pointes in patients with acquired prolonged repolarization: quantitative analysis of Holter recordings. $7 \mathrm{Am}$ Coll Cardiol 1995;25:1564-75.

15 Sutko JL, Bers DM, Reeves JP. Postrest inotropy in rabbit ventricle: $\mathrm{Na}^{+}-\mathrm{Ca}^{2+}$ exchange determines sarcoplasmic reticulum $\mathrm{Ca}^{2+}$ content. Am $\mathfrak{f}$ Physiol 1986;250:H654-61.

16 Bers DM, Bassani RA, Bassani JW, et al. Paradoxical twitch potentiation after rest in cardiac muscle: increased fractional release of SR calcium. $\mathcal{F}$ Mol Cell Cardiol 1993;25:1047-57.

17 El-Sherif N, Caref EB, Chinushi M, et al. Mechanism of arrhythmogenicity of the short-long cardiac sequence that precedes ventricular tachyarrhythmias in the long QT syndrome. F Am Coll Cardiol 1999;33:1415-23.

\section{Electronic pages}

\section{eHEART: www.heartjnl.com}

The following electronic only articles are published in conjunction with this issue of Heart.

\section{Pressure damping, a "billowing" septum, and an eerie silence: perioperative, intermittent obstruction of a mitral valve prosthesis \\ W Keeble, $S$ M Cobbe}

This case involving a 74 year old man who underwent mitral valve and aortic valve replacements provides detailed insight into the perioperative echocardiographic and haemodynamic changes occurring when a mitral valve prosthesis intermittently obstructs. It illustrates the early sequence of electromechanical dissociation which would lead to cardiac arrest should a tilting disc prosthesis be immobilised in the closed position.

(Heart 2000;84:e6) www.heartjnl.com/cgi/content/full/84/ 3/e6
Coexistence of giant aneurysm of sinus of Valsalva and coronary artery aneurysm associated with idiopathic hypereosinophilic syndrome

\section{T Okinaka, N Isaka, T Nakano}

Aneurysms of the coronary sinuses of Valsalva and coronary artery aneurysms are uncommon cardiac anomalies, and cases in which these two uncommon lesions occur at the same time are extremely rare. A case of a woman with unstable angina who had a giant aneurysm of the left coronary sinus and multiple coronary artery aneurysms associated with an idiopathic hypereosinophilic syndrome is presented. Her sustained eosinophilia, elevated eosinophilic cationic protein concentration, and pathological findings of eosinophil infiltration of the aortic wall suggested the association of eosinophilia induced vascular injury as the cause of these aneurysms. This is the first such case to survive following surgical treatment.

(Heart 2000;84:e7) www.heartjnl.com/cgi/content/full/84/ 3/e7 